\title{
Canceling evanescent waves in high-energy superconducting rf linacs
}

\author{
Vladimir N. Litvinenko \\ Brookhaven National Laboratory, Upton, New York 11973, USA
}

(Received 23 December 2009; published 11 May 2010)

\begin{abstract}
Many future projects plan using high-current, high-energy, multipass energy-recovery linacs that are based on superconducting rf (SRF) cavities. The necessity of ensuring the transverse stability of the beam in such accelerators imposes strict limits on the high order modes (HOMs) impedances, and demands effective HOM damping. The latter requirement often precludes achieving a high real-estate accelerating gradient in such structures. The modular structure of long SRF linacs also requires incorporating vacuum flanges; however, these flanges have surface contacts and cannot tolerate strong rf fields. Locating them in the low-field areas of the linac structure may involve considerably elongating the intermodular interfaces, a change that would reduce the linac's real-estate accelerating gradient. In this paper, I propose a novel method to resolve this issue, using compact interconnects between the SRF cavities wherein to locate effective HOM dampers and vacuum flanges.
\end{abstract}

DOI: 10.1103/PhysRevSTAB.13.051001

PACS numbers: 29.20.Ej, 85.25.- j

\section{INTRODUCTION}

Several proposals for electron-hadron colliders and light sources [1-15] and advanced electron coolers [16-19] are based on superconducting energy-recovery linacs (SRF ERLs) operating with very high average currents. Some such projects propose using multipass ERLs to mitigate the cost of their SRF linacs. High-energy SRF linacs are comprised of many multicell SRF cavities separated by transitions, which I will call interconnects. SRF cavities are notorious for the very high quality factor, $Q \sim$ $10^{9}-10^{10}$, of its modes. The high $Q$ of high order modes (HOMs) entails very high impedances and the corresponding low thresholds for beam instabilities in an ERL. Particularly, the need for transverse stability of electron beam [20-23] is known to impose very strong restrictions on the impedances and $Q$ s of dipole HOMs. Recent studies showed that high- $Q$ quadrupole HOMs also may endanger the stable operation of ERLs. In practice, this means that SRF cavities should not have trapped dipole HOMs, and possibly no quadrupole ones. In addition, all HOMs should be strongly damped, either by absorbers or loaded HOM couplers. Furthermore, the HOM absorbers should not significantly affect the $Q$ of the fundamental mode (FM), i.e., the monopole mode accelerating and decelerating electrons. Indeed, one of the foremost reasons for using SRF linacs lies in their low losses that are related directly to the very high $Q$ of the FM. Second, strong coupling of the HOM absorbers to the FM power would assure that they absorb a very large amount of power, which should be both generated and evacuated. Accomplishing the latter is not a trivial task.

Cooling ceramic or ferrite high-power absorbers at cryogenic temperatures is complicated and expensive, even if it can be done [24]. Using HOM pickup probes equipped with a notch filter at the FM frequency $[25,26]$ very effec- tively damps HOMs without adversely affecting the FM. Nevertheless, experience with the Spallation Neutron Source (SRF) linac demonstrated the limitations of this technology, suggesting that such HOM couplers should not be placed into strong FM fields [27].

In short, to approach the goal of high-current ERLs, the HOM absorbers must be strongly decoupled from the FM field, while being strongly coupled to all HOMs of interest.

One possible design, proposed by Rimmer et al., is using waveguides with a cutoff frequency above that of the FM to transport the HOM power to the room-temperature HOM loads [28]. Using six HOM waveguides per cavity, as they suggested, significantly complicates the design, and requires having six additional large transitions from $2 \mathrm{~K}$ to room temperature. While their approach is very attractive, it may lead to more expensive cavities, and significantly increase the consumption of liquid helium to cool multiple waveguides extending from cryogenic to room temperatures.

Cost and size considerations for SRF accelerators require a high real-estate accelerating gradient and, therefore, tightly packed SRF cavities. Figure 1 shows the typical layout of a long multicavity SRF linac.

The real-estate (RE) accelerating gradient of such a linac is

$$
E_{\mathrm{RE}}^{\prime}=E_{c}^{\prime} \frac{N \cdot l_{c}}{N \cdot\left(l_{c}+l_{i}\right)+2 l_{t r}-l_{i}},
$$

where $E_{c}^{\prime}$ is the cavity's accelerating gradient, $l_{c}$ is the cavity's length, with $l_{t r}, l_{i}$, respectively, being the lengths of the room- to-cryogenic temperature transitions, and the cavity interconnects. For a large number of cavities, $N \gg$ 1 , the scaling is straightforward

$$
E_{\mathrm{RE}}^{\prime} \approx E_{c}^{\prime} \frac{l_{c}}{l_{c}+l_{i}},
$$




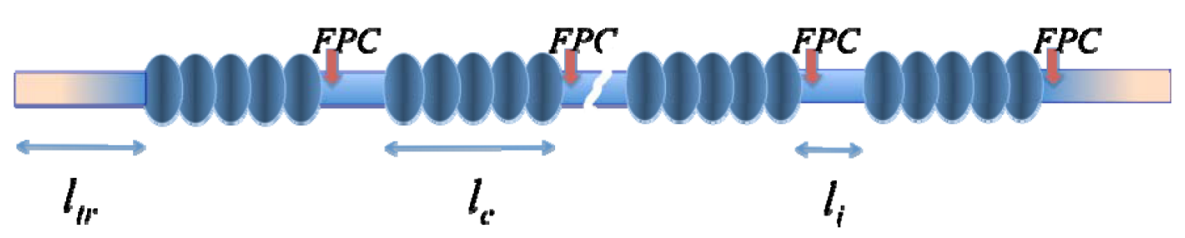

FIG. 1. (Color) A schematic of a high-current, multicavity SRF linac, with fundamental power couplers (FPCs). It comprises $N$ fivecell cavities linked by interconnects, and terminated by cryogenic-to-room-temperature transitions.

i.e., shortening the interconnect is the most effective way of increasing the linac's real-estate gradient and reducing its cost.

In Sec. II, I will consider, for simplicity, interconnects in the shape of uniform circular tubes. Analytical solutions for more complicated interconnects with nonuniform cross sections or fluted waveguides [29] do not exist and, therefore, are less illustrative. The applicability of the proposed method to interconnects with a different geometry is discussed later.

\section{EVANESCENT WAVE IN THE INTERCONNECTING TUBE}

The rf wavelength of the linac's FM $\lambda_{\mathrm{FM}}=2 \pi / k_{\mathrm{FM}}$ sets a natural scale for the length of the connections. I will express the length of the tubes in these units, i.e., the unitlength tube will be a tube with $l_{i}=\lambda_{\mathrm{FM}}$.

Traditionally, the FM field is suppressed in the interconnects with a cutoff frequency above the FM frequency, $\omega_{\mathrm{FM}}=k_{\mathrm{FM}} c$ :

$$
\omega_{\text {cutoff }}=\frac{c}{r_{o}} \cdot x_{1} \cong 2.40482 \cdot \frac{c}{r_{o}}
$$

where $c$ is the speed of the light, $r_{o}$ is by the tube's radius, and $x_{1}$ is the first root of the zeroth-order ordinary Bessel function: $J_{o}\left(x_{1}\right)=0$. In this case, the FM field for the dominant $\mathrm{TM}_{01}$ monopole mode decays as an evanescent wave in a waveguide [30]:

$$
\begin{aligned}
E_{z}(z, t) & =E_{1} \cdot e^{-\kappa_{1} \cdot\left|z-z_{o}\right|} e^{i\left(\omega_{\mathrm{FM}} t+\varphi_{o}\right)}, \\
\kappa_{1} & =k_{\mathrm{FM}} \sqrt{\left(\frac{x_{1}}{k_{\mathrm{FM}} r_{0}}\right)^{2}-1}
\end{aligned}
$$

where $E_{1}$ is the amplitude, and $\varphi_{0}$ is the phase of the longitudinal electric field at the beginning of the interconnecting tube located at $z_{o}$. I note that the evanescent wave has the same phase through the tube.

Expressing this in more detail, the transverse structure of the FM in the cavity differs from that in the tube; that is, at the end of the cavity the FM field couples with a number of higher order TM and TE monopole modes in the tube:

$$
\begin{aligned}
E_{z}^{\mathrm{TM}}(r, z, t)= & \sum_{m=1}^{\infty} E_{m} \cdot J_{o}\left(\frac{r}{r_{o}} x_{m}\right) \cdot e^{i\left(\omega_{\mathrm{FM}} t+\varphi_{\mathrm{TE}}\right)} \\
& \cdot e^{-\kappa_{m}^{\mathrm{TM}} \cdot\left|z-z_{o}\right|} ; \\
H_{z}^{\mathrm{TE}}(r, z, t)= & \sum_{m=1}^{\infty} H_{m} \cdot J_{o}\left(\frac{r}{r_{o}} x_{m}^{\prime}\right) \cdot e^{i\left(\omega_{\mathrm{FM}} t+\varphi_{\mathrm{TM}}\right)} \\
& \cdot e^{-\kappa_{m}^{\mathrm{TE}} \cdot\left|z-z_{o}\right|} \\
\kappa_{m}^{\mathrm{TM}}= & k_{\mathrm{FM}} \sqrt{\left(\frac{x_{m}}{k_{\mathrm{FM}} r_{0}}\right)^{2}-1} ; \\
\kappa_{m}^{\mathrm{TE}}= & k_{\mathrm{FM}} \sqrt{\left(\frac{x_{m}^{\prime}}{k_{\mathrm{FM}} r_{0}}\right)^{2}-1},
\end{aligned}
$$

where $x_{m}$ is the $m$ th root of the zeroth-order Bessel function: $J_{o}\left(x_{m}\right)=0 ; \quad x_{1} \cong 2.40482, \quad x_{2} \cong 5.520, \quad x_{3} \cong$ $8.654 \ldots$, while $x_{m}^{\prime}$ is the $(m+1)$ th root of its derivative: $J_{o}^{\prime}\left(x_{m}^{\prime}\right)=0 ; x_{1} \cong 3.832, x_{2} \cong 7.016 \ldots$ [30]. Because the higher order monopole modes decay very quickly in the tube, only the dominant $\mathrm{TM}_{01}$ monopole mode plays a practical role in the designs of SRF linacs. Indeed, in a unit-length tube, the amplitude $\mathrm{TM}_{02}$ mode decays by about six orders of magnitude, while the $\mathrm{TM}_{03}$ mode decays in excess of nine orders of magnitude. The amplitude of the $\mathrm{TE}_{01}$ mode also is reduced by at least 2500-fold at this distance. Accordingly, at $\left|z-z_{0}\right| \sim \lambda_{\mathrm{FM}}$ the FM power of the TM modes with $m \geq 2$ is significantly smaller than rf power losses in the SRF cavity and does not present any problems.

Similarly, the higher multipole $\mathrm{TM}_{m n}$ and $\mathrm{TE}_{m n}$ modes, such as dipole, sextupole, and the like, at FM frequency can be generated by asymmetries in the system (such as the FPC), but they also decay much faster than the $\mathrm{TM}_{01}$ mode. The exception here is the $\mathrm{TE}_{11}$ mode with a cutoff frequency of $\omega_{\mathrm{co} \mathrm{TE}_{11}} \cong 1.841 \cdot r_{o} / c$ [30]. Therefore, additional precautions may be needed to avoid exciting this FM component in the waveguide, for example, using axissymmetric FPCs.

\section{THE BNL FIVE-CELL SRF CAVITY}

Suppressing the dominant $\mathrm{TM}_{01}$ monopole $\mathrm{FM}$ in the pipe strongly depends on the choice of the cutoff frequency. Figure 2 depicts the structure of the five-cell 

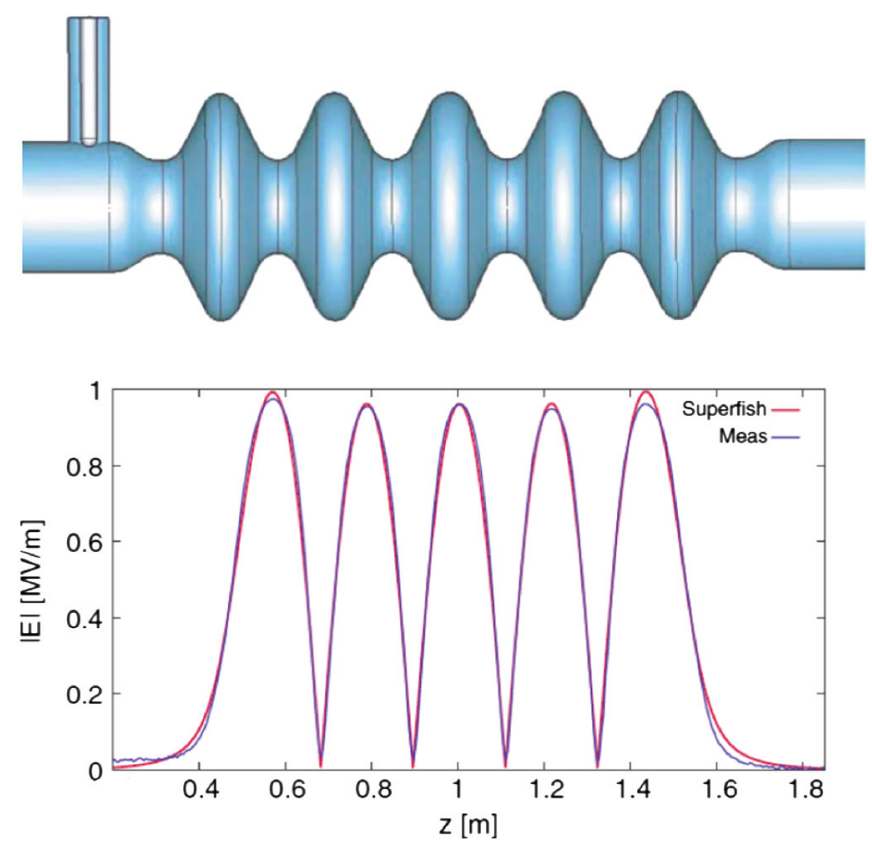

FIG. 2. (Color) Structure of the $703 \mathrm{MHz}$ BNL five-cell cavity (top), and a simulated by SUPERFISH and a measured field envelope of the fundamental mode (bottom) [31].

$703 \mathrm{MHz}$ SRF cavity designed and built at BNL with $r_{o}=$ $12 \mathrm{~cm}$ tubes. These tubes have a cutoff frequency of $956 \mathrm{MHz}$ for the $\mathrm{TM}_{01}$ mode, $732 \mathrm{MHz}$ for the $\mathrm{TE}_{11}$ mode, and $1.554 \mathrm{GHz}$ for $\mathrm{TM}_{11}$ [31]. The bottom graph in Fig. 2 shows the FM field envelope in the cavity, and its rapid decay in the tubes.

Such a low cutoff frequency is used to propagate the fields of the troublesome dipole modes towards the ferrite absorbers located at the end of the pipes. BNL's cavity has two low-frequency dipole passbands: $\mathrm{TE}_{11}$ modes in the $0.75-0.9 \mathrm{GHz}$ frequency range, and $\mathrm{TM}_{11}$ modes in the 0.95-1.0 GHz frequency range [32]. Thus, the low $\mathrm{TE}_{11}$ cutoff frequency of $732 \mathrm{MHz}$ ensures a very good propagation of the dipole TE modes towards the absorbers. In contrast, a very high $\mathrm{TM}_{11}$ cutoff frequency of $1.554 \mathrm{GHz}$ does not support the effective coupling of the dipole TM modes to the absorbers. Since these modes are responsible for transverse beam breakup instabilities, the TM dipole modes are the most harmful ones, and damping them is of critical importance.

The cavity dipole TM modes (in this band) are coupled with the absorbers through their partial conversion into the tube (waveguide) $\mathrm{TE}_{11}$ modes at the end of the cavity [3335]. Thus, assuring the effectualness of this conversion is essential for proper damping of these modes.

In BNL's R\&D five-cell SRF cavity, two ferrite absorbers are located outside the cryostat, viz. at room temperature, and are about $1 \mathrm{~m}$ away from the ends of the cavity. In these long pipes, the $\mathrm{TM}_{01} \mathrm{FM}$ power drops by about 12 orders of magnitude, and only milliwatts of rf power escape the pipes and reach the HOM absorbers.
Further in the paper, I use the parameters of five-cell BNL SRF cavity for numerical comparisons, specifically, the ratio between the pipe's $\mathrm{TM}_{01}$ cutoff frequency, and the FM frequency of $R=\omega_{\text {cutoff }}^{\mathrm{TM}_{01}} / \omega_{\mathrm{FM}}=1.36$.

\section{EFFECTIVE MULTICELL HOM-DAMPED SRF LINACS}

Measurements of BNL's R\&D five-cell SRF cavity demonstrated that all dangerous dipole HOMs could be damped to the level necessary for the ampere-class ERLs [32]. At the same time, such long tubes, together with about a meter-long five-cell cavity, are unsuitable for high-energy linacs because of a very low real-estate gradient. Increasing the length of the cavity by incorporating more than five cells might result in trapped modes with very high $Q$ s that are unacceptable in high-current ERLs.

A cost-effective resolution for this problem lies in reducing the length of interconnecting tubes. The length of the SRF cavity cell in a high-energy linac is very close to half that of the rf wavelength, $l_{\text {cell }} \cong \lambda_{\mathrm{FM}} / 2$. The length of an HOM- damped five-cell cavity will be $\sim 2.5$ units: $l_{c} \cong$ $2.5 \lambda_{\mathrm{FM}}$. While the five-unit-long tube's equivalent used in the BNL's R\&D cavity functions satisfactorily, for highenergy linacs, a threefold lowering of the real-estate gradient compared with that in the cavity is not an acceptable solution. Using a one-unit-length tube would degrade the linac's real-estate gradient by about $30 \%$. A linac with twounit-long tubes would approximately halve the gradient.

Here I discuss the attractive option of a unit-length tube, wherein the HOM absorbers (or HOM pickups) and the vacuum flange are located near its center. This configuration means that absorbing units are about $1 / 2$ of the unit from the end of the cavity. For a FM frequency $\sim 1 \mathrm{GHz}$, and end-of-the-cavity opening radius $\sim 5 \mathrm{~cm}$, a cavity with $20 \mathrm{MV} / \mathrm{m}$ accelerating gradient could couple $\sim 200 \mathrm{MW}$ of $\mathrm{rf}$ power into the tube if it is properly terminated. ${ }^{1}$ Using a tube with $R=1.36$ provides for a 325 -fold reduction of the rf power at the $1 / 2$ unit length, and a $10^{5}$-fold reduction at the entrance of the neighboring cavity.

Consequently, $\sim 1 \mathrm{~kW}$ of rf power may leak from one cavity to another, an amount that is significantly smaller than the typical transmitter power of $\sim 10 \mathrm{~kW}$ required to compensate for microphonics in such an SRF cavity. A proper rf feedback system can overpower such cross talking between the cavities, and maintain the desirable accelerating voltage and phase independently in each cavity.

\footnotetext{
${ }^{1}$ In the SRF linacs and interconnecting tubes under discussion in this paper, the fields are represented by standing waves. There is a very small amount of propagating power in the system, when compared with the enormous reactive power stored in the cavity. Here I quote rf power as a measure of power, which could be delivered if the system is terminated by a proper (matched) resistive load. In other words, this is the maximum power, which could be drained of by an absorber.
} 


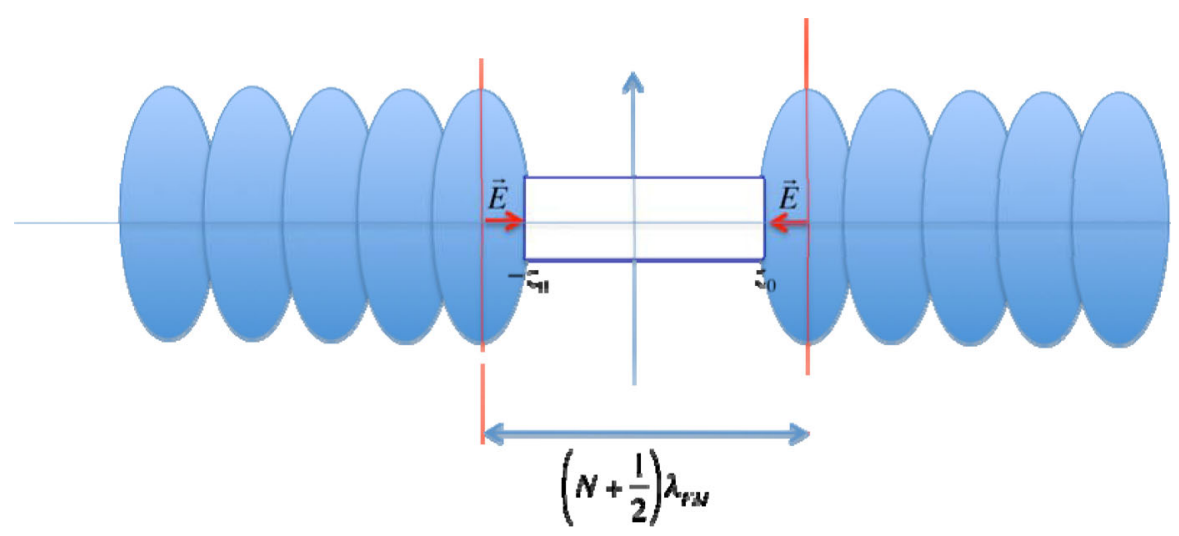

FIG. 3. (Color) Placing two cavities about $(2 N+1) \lambda_{\mathrm{FM}} / 2$ apart, where $N$ is an integer, provides their natural phasing of 180 degrees apart, and the opposite sign of the electric field in their end cells.

In contrast, the vacuum flange and the HOM absorbers might be exposed to $\sim 1 \mathrm{MW}$ rf power and an $\mathrm{rf}$ electric field $\sim 0.5 \mathrm{MV} / \mathrm{m}$. This level of rf power can cause major heat losses in the flanges and the HOM absorbers, and potentially might damage the latter.

\section{CANCELING EVANESCENT FM}

I propose using the natural cancellation of the evanescent waves (4) from two adjacent cavities, by selecting the distance between them such that the evanescent waves at opposite ends of the tube have the same phase but the opposite sign of the electric field's $z$ component. Figure 3 shows an approximate configuration of the system. For

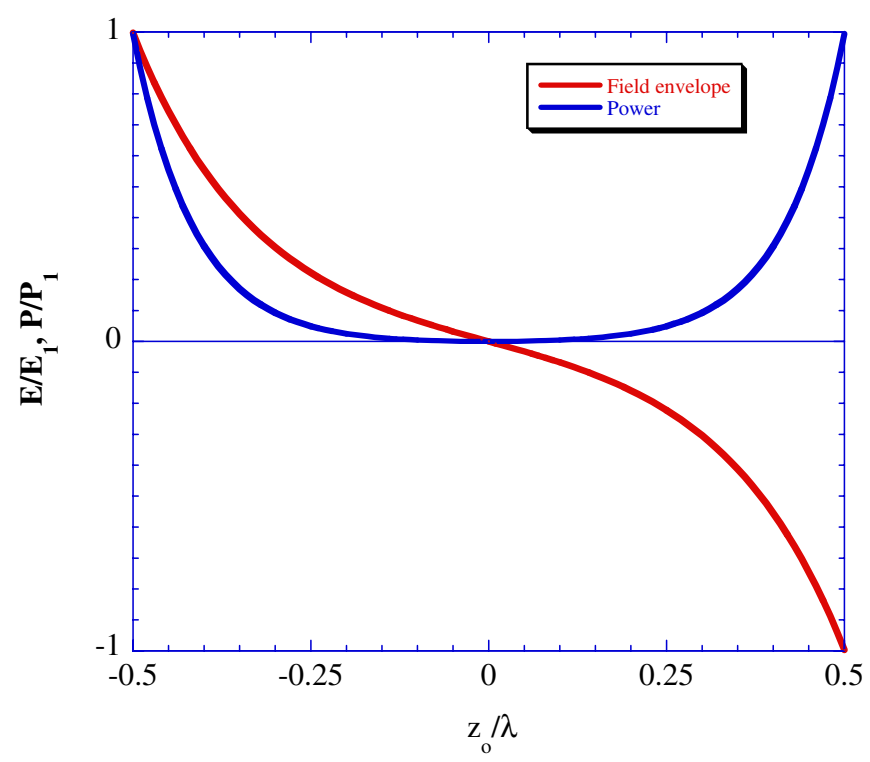

FIG. 4. (Color) Normalized field envelope (red) and the power envelope (blue) for a unit-length tube with cancellation of the evanescent waves. The field and the power are normalized to $E_{1}$ and $P_{1}$, correspondingly. This graph uses the unit-length tube $\left(l_{i}=\lambda_{\mathrm{FM}}\right)$, and the ratio in BNL's R\&D five-cell cavity between the cutoff and FM frequencies $\left(\kappa_{1} \cdot \lambda_{\mathrm{FM}}=5.79\right)$. simplicity, I consider here two cavities having equal FM fields. Deviations from this assumption are discussed in the next section.

In such a configuration, the evanescent waves cancel each other out in the middle of the tube:

$$
\begin{gathered}
\tilde{E}_{z}(z, t)=2 E_{1} \cdot e^{-\kappa_{1} z_{o}} \sinh \left(\kappa_{1} z\right) \cdot e^{i\left(\omega_{\mathrm{FM}} t+\varphi_{0}\right)} \\
z_{0}=N \lambda_{\mathrm{FM}} / 2 ; \quad \tilde{P}(z)=4 P_{1} \cdot e^{-2 \kappa_{1} z_{o}} \sinh ^{2}\left(\kappa_{1} z\right),
\end{gathered}
$$

where $P_{1}$ is the power of the FM at the entrance of the tube. ${ }^{2}$ Figure 4 shows the field envelope and the rf power as a function of the coordinate along the tube. The FM field is zero in the center of the tube, and is an ideal place for putting potentially lossy elements, such as flanges and HOM absorbers. What is remarkable in such a configuration is that the power level grows as the second power of the distance from the center $\left(P \sim z^{2}\right)$, and that the power in about $30 \%$ of the pipe $\left(\sim 0.3 \lambda_{\mathrm{FM}}\right)$ is below $1 \%$ of the $P_{1}$.

Figure 5, where power is plotted on a logarithmic scale, demonstrates the effect of canceling the evanescent waves by comparing it to the case when the fields at the end of the tube are in phase.

This practical example shows that about 0.1 of unit length (4 cm for the $703 \mathrm{MHz}$ SRC cavity) has a tenfold reduction of the power, and 3\% of its length suppresses power a hundredfold.

Thus, canceling the evanescent waves additionally reduces by one-to-two orders of magnitude the FM power at the location of absorbing elements. For the vacuum-flange connections, this power reduction greatly simplifies the design and increases the number of options.

In combination with other means of rejecting FM, canceling these waves could effectively damp HOMs with very low loss or none in the FM $Q$ factor. HOM couplers/absorbers placed in the middle of a tube with evanescent wave canceling are exposed to a much lower FM field, and, therefore, their operation will be more robust.

\footnotetext{
${ }^{2}$ See footnote on previous page.
} 


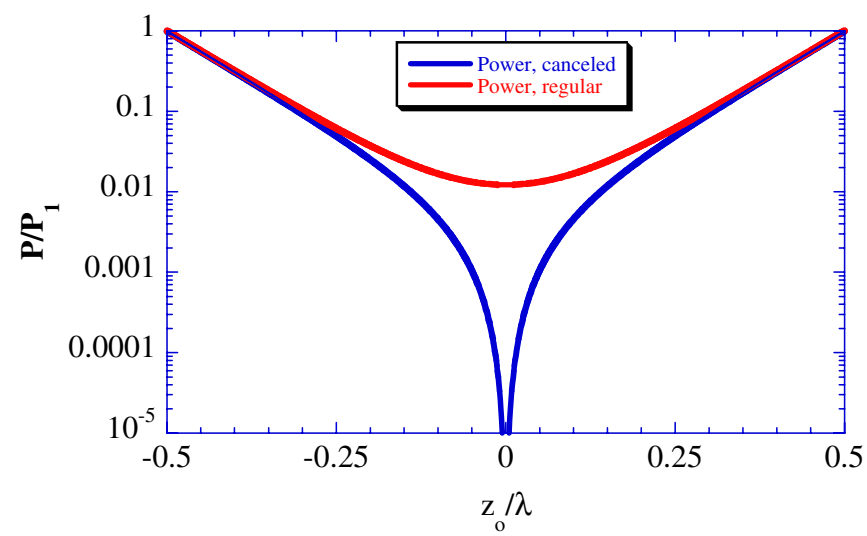

FIG. 5. (Color) Power distribution inside a unit-length tube. (a) Blue curve - the power of canceling evanescent waves with $(2 n+1) \pi$-phase difference, and (b) red curve-the phased evanescent waves with a $2 n \pi$ phase difference.

Such HOM couples can either use FM rejection filters $[25,26]$ or geometrical factors.

\section{SENSITIVITY OF ERRORS}

In practice, several factors might affect the cancellation of evanescent waves; neighboring cavities may have different accelerating gradients and/or phases. The FPCs also can affect the evanescent wave.

Thus, I start by considering the asymmetric effects, such as an FPC that, by design, is located close to its home cavity. The FPC may modify the amplitude and, possibly, the phase of the $\mathrm{TE}_{01}$ evanescent wave coming from its home cavity:

$$
E_{z}(z, t)=\left(E_{1}+\Delta E\right) \cdot e^{-\kappa_{1} \cdot\left|z-z_{o}\right|} e^{i\left(\omega_{\mathrm{FM}} t+\varphi_{o}+\Delta \varphi\right)} .
$$

Since software can simulate both the change in amplitude and the phase changes, the phase error could be corrected by adjusting the length of the tube, setting the phase difference at $180^{\circ}$. The difference in the amplitudes at the opposite sides of the tube will shift the node in the FM field and power. In general, in a tube with FM TE 10 amplitude, $E_{1}$ at $z=-z_{0}$ and $E_{2}$ at $z=z_{0}$, the envelope of the field is obtained by a simple modification of Eq. (6):

$$
\begin{gathered}
E_{z}(z, t) e^{-i\left(\omega_{\mathrm{FM}} t+\varphi_{o}\right)}=2 E \cdot e^{-\kappa_{1} z_{o}} \cdot \operatorname{sh}\left[\kappa_{1}(z-\hat{z})\right] ; \\
E=\sqrt{E_{1} E_{2}} ; \quad \hat{z}=\ln \left(E_{1} / E_{2}\right) / 2 \kappa_{1} .
\end{gathered}
$$

Consequently, in a properly designed linac, the absorbing elements should be located at $\hat{z}=\ln \left(E_{1} / E_{2}\right) / 2 \kappa_{1}$.
More general variations of the boundary conditions can arise either from manufacturing errors or from running the linac with different fields in the cavities. I return again to the generic linac, depicted in Fig. 1, that is comprised of $N$ cavities. Such a linac would have $N$ rf transmitters connected to its home cavity FPC, and low-level rf feedback systems to maintain desirable accelerating rf voltages (i.e., the FM $E$ field) and phases in each cavity. The majority of the power in such an rf transmitter is used to combat microphonic effects, that is, fast variations in the cavity's frequency caused by mechanical vibrations. Here, I reasonably assume that power leakage from one cavity to another is significantly smaller than available rf power from the FPCs, and that the control system can maintain the designed accelerating voltage and phase in each of them.

Therefore, in all cavities the FM would have identical frequency, ${ }^{3}$ with fields phase locked to the master oscillator of an accelerator. Being a system of $\mathrm{N}$-driven oscillators, any state of the FM field of such linac can be represented as the sum of the $N$ modes:

$$
E(n)=\operatorname{Re} \sum_{n=0}^{N-1} a_{n} \cdot e^{i\left[\omega_{\mathrm{FM}} t+2 \pi(n / N)\right]},
$$

where $n$ is the cavity number in the linac, and the cavity phase is related to the center of the accelerating beam.

If all cavities have identical accelerating fields and identical (zero) phase, then all modes would vanish, except the $n=0$ in-phase mode. If the accelerating fields and phases in the cavities vary, there will contributions from $n \neq 0$ modes in Eq. (9).

In the typical case of two cavities, which is often discussed [36], there are two modes; the in-phase and the outof-phase one (the so-called $\pi$ mode). In this specific case of the two phase-locked cavities with the same accelerating field would have zero amplitude of the $\pi$ mode. In other words, the amplitude of $\pi$ mode is directly proportional to the difference in the accelerating field of the two cavities.

The preceding discussion established the fact that considering $N$ cavities with various accelerating fields and phases is equivalent to considering all $2 \mathrm{~N}$ modes in the coupled oscillator's frame of reference. Hence, I next look at the most general case, that of two neighboring cavities differing from the ideal case both in the amplitude and the phase at the ends of the connecting tube:

$$
-E_{1} e^{-\kappa_{1}\left(z+z_{o}\right)} e^{i\left(\omega_{\mathrm{FM}} t+i \varphi_{1}\right)} ; \quad E_{2} e^{\kappa_{1}\left(z-z_{o}\right)} e^{i\left(\omega_{\mathrm{FM}} t+i \varphi_{2}\right)} .
$$

The resulting field in the tube readily is found as

\footnotetext{
${ }^{3}$ I note that this is completely different from an assemble of $N$ freestanding cavities that oscillate on their own frequency
} 
TABLE I. Effect of errors on canceling evanescent waves.

\begin{tabular}{lcc}
\hline \hline & Effect in the field & Effect in power \\
\hline Phase error & $\delta\left|E_{z}\right|(0)=2 E_{1} e^{-\kappa_{1} z_{o}} \cdot \sin \Delta \varphi$ & $\delta P(0) \propto 2 P_{1} e^{-2 \kappa_{1} z_{o}} \cdot \sin ^{2} \Delta \varphi$ \\
Error in the amplitude & $\delta\left|E_{z}\right|(0)=e^{-\kappa_{1} z_{o}} \cdot\left(E_{1}-E_{2}\right)$ & $\frac{\delta P(0)}{P_{1} \cdot e^{-2 \kappa_{1} z_{o}}}=\left(\frac{E_{1}-E_{2}}{E_{1}+E_{2}}\right)^{2}$ \\
Position error/final length of the probe & $\left|E_{z}\right|=e^{-\kappa z_{o}} \cdot 2 E_{o} \cdot \sinh \kappa_{1} z$ & $P=2 P_{1} e^{-2 \kappa z_{o}} \cdot \sinh ^{2} \kappa_{1} z$ \\
\hline \hline
\end{tabular}

$$
\begin{aligned}
& E_{z}=e^{-\kappa_{1} z_{o} \cdot \operatorname{Re}^{i\left(\omega_{\mathrm{FM}} t+\varphi\right)}\left(\begin{array}{l}
\cos \Delta \varphi \cdot\left[\cosh \kappa_{1} z \cdot\left(E_{1}-E_{2}\right)-\sinh \kappa_{1} z \cdot\left(E_{1}+E_{2}\right)\right] \\
+i \sin \Delta \varphi \cdot\left[\cosh \kappa_{1} z \cdot\left(E_{1}+E_{2}\right)-\sinh \kappa_{1} z \cdot\left(E_{1}-E_{2}\right)\right]
\end{array}\right)} \\
& \varphi=\frac{\varphi_{1}+\varphi_{2}}{2} ; \quad \Delta \varphi=\frac{\varphi_{1}-\varphi_{2}}{2} .
\end{aligned}
$$

This general equation allows us to study the effect of all possible errors; Table I summarizes them. As is evident, differences between the two cavities in phase up to $10 \mathrm{de}$ grees and in amplitude (accelerating gradients) up to $20 \%$ still provide hundredfold suppression in evanescent wave power.

The error in the longitudinal positioning of the absorbing elements also is relaxed; Figs. 4 and 5 clearly demonstrate this trend. In the $703 \mathrm{MHz}$ SRF linac, an absorbing element having a $0.5 \mathrm{~cm}$ positioning error still would experience about a 250-fold suppression of FM power.

Hence, conditions for cancellation of the FM evanescent waves in the interconnecting tubes are relaxed, and do not require exceptionally high precision both in tuning the linac and in positioning the absorbing elements.

For further discussions let us define efficiency of evanescent wave cancellation, $R$, by the following expression:

$$
1 / R=\frac{\left|E_{1} e^{-\kappa_{1} z+i \Delta \varphi}-E_{2} e^{\kappa_{1} z-i \Delta \varphi}\right|^{2}}{4 E_{o}^{2}},
$$

where $E_{o}$ is the maximum design value of the electric field at the entrance of the entrance of the tube. In large-scale SRF installations, some of the cavities have to be operated at reduced power. As shown above, the cancellation scheme would provide a 16-fold reduction of the FM power in the location of HOM absorber, when accelerating gradient in one of the cavities is reduced by $50 \%$ in one of the cavities.

In the worst-case scenario, when one of the cavities has to be turned off by a technical reason and no other measures are taken, the cancellation of the evanescent waves is off in the HOM dampers at both ends of this cavity, and they will be exposed to $1 / 4$ of the FM rf power $(R=4)$. If a loss of single cavity (or multiple cavities) is an acceptable operating scenario for such a facility, then the situation can be mitigated by gradual reduction of the accelerating gradients in the neighboring cavities. For example, setting two neighboring cavities at $50 \%$ of the nominal accelerating will provide 16-fold reduction of FM because of partial cancellation of the FM waves [Eq. (11) and Table I, line 2].
The end-of-the-linac cavity cannot use cancellation of evanescent waves at one side of the cavity. Hence, the length of transitions $\left(l_{r t}\right)$ should be sufficient for evanescent wave decay to an acceptable level for HOM absorber. This would result in modest reduction of the real-estate gradient if linac is composed of many cavities, i.e. $N \gg 1$ [see Eq. (1)].

\section{PULSED MODE OF OPERATION AND TRANSIENTS}

In the previous section we discussed issues relevant to cw SRF linacs. In a pulsed SRF linac with instant turning on of an rf transmitter, a transient from zero to maximum field $E_{o}$ (or power $P_{o}$ ) is described as follows:

$$
\begin{gathered}
P=P_{o}\left(1-e^{-t / \tau}\right) ; \quad E=E_{o}\left(1-e^{-t / 2 \tau}\right) ; \\
\tau=\frac{Q_{L}}{\omega_{\mathrm{FM}}},
\end{gathered}
$$

where $Q_{L}$ is loaded $Q$ factor, which can slightly vary from a cavity to a cavity. The left graph in Fig. 6 shows evolution of the electric fields in three cavities with a typical $\pm 10 \%$ variation in $Q_{L}$. Turning the cavity off is equivalent to turning the graph upside down: $P=P_{o} e^{-t / \tau}$. The right graph shows the efficiency of the evanescent wave cancellation during such transitions.

As can be seen from Fig. 6, the fields do not deviate significantly and evanescent wave cancellation is next to perfect ( $R \sim$ few thousands). It is quite remarkable that $50 \%$ deviation in $Q_{L}$ still provides $R \sim 200$, while a factor 2 variation in $Q_{L}$ would result in $R>60$. Hence, reasonable spread of loaded $Q$ factors does not significantly affect the cancellation.

In contrast, the phase transients with phase variations between neighboring cavities $\sim 20$ degrees and above can be harmful. A proper phase feedback system is required to avoid such significant phase swings or potential benefits of evanescent wave cancellation would be lost.

Finally, even cw linacs also should be turned on and turned off. In this case, the rf power can be brought up and 

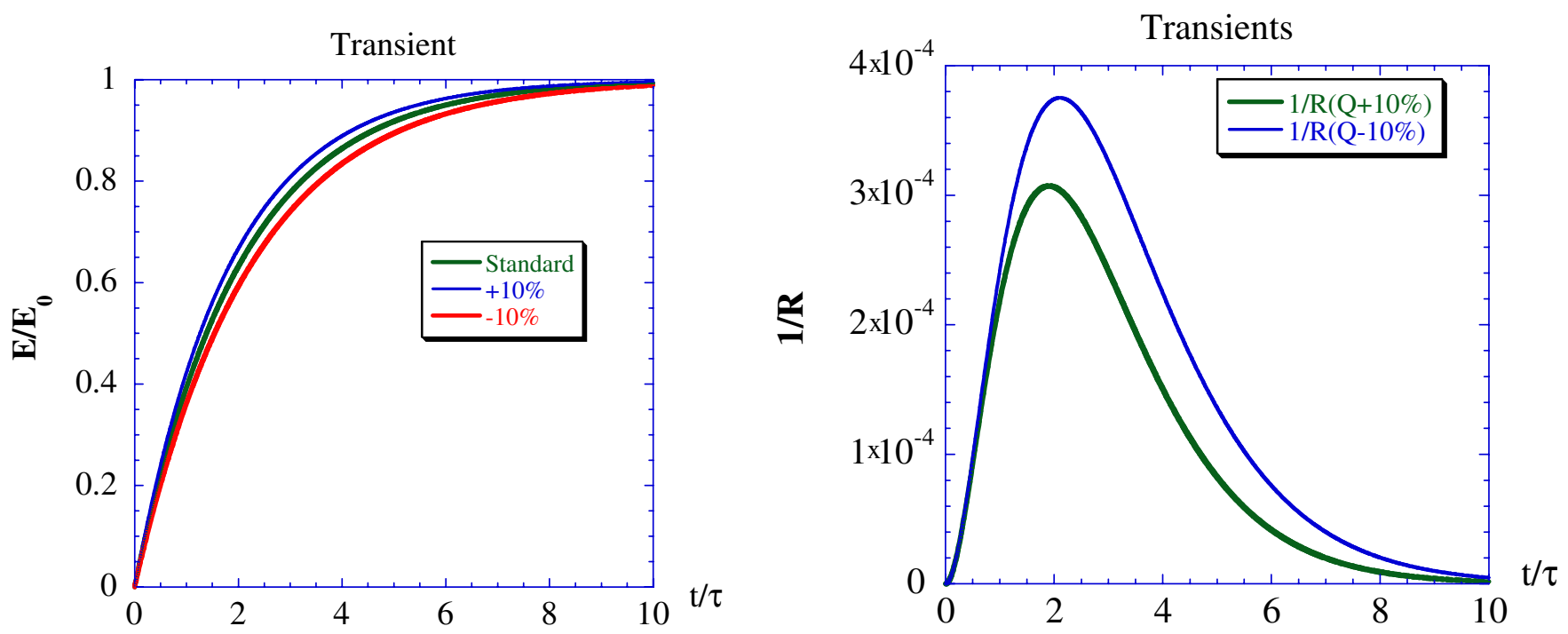

FIG. 6. (Color) Transient of the electric fields (left graph) and the efficiencies of evanescent wave cancellation during turning-on cavities with $\pm 10 \%$ variation of $Q_{L}$.

turned off slowly in a controlled manner and the cancellation conditions could be preserved.

\section{DISCUSSION}

As I demonstrated in the previous sections, a multicavity SRF linac with unit-length interconnecting tubes wherein evanescent waves are canceled can exhibit a very high $Q$ (low loss) of the FM, and very effective HOM damping. This especially is true for the most dangerous dipole HOMs.

One important question that was not discussed in the previous section is the possibility of cancellation of the HOM amplitudes at the location of the HOM-absorbing elements. In contrast to the FM frequencies of the cavities, which are driven into the exact synchronism by the feedback system, the HOM frequencies of cavities generally do not coincide. Hence, cancellation of HOMs from two cavities is practically impossible, or at least improbable.

Using interconnecting tubes with a variable profile or/ and a different cross section may modify the mode structure and add additional mode-to-mode transformation. Nevertheless, the FM must decay in such structures (i.e., be an evanescent wave), and, indeed, the cancellation of two contrapropagating waves might be possible in such structures.

\section{CONCLUSIONS}

The proposed cancellation of the evanescent waves at fundamental cavities offers a means of achieving major additional reduction in the power of the fundamental mode to which the absorbing elements of SRF linac structure (flanges and HOM absorbers) are exposed. In combination with other elegant FM rejection techniques, such as HOM pickup probes equipped with a notch filter at the FM frequency, this method can ensure compact, effective HOM-absorbing structures, and, practical high-current ERLs with high real-estate accelerating gradient linacs.

\section{ACKNOWLEDGMENTS}

I am grateful to Ilan Ben-Zvi and Harald Hahn (BNL) for thoughtful, in-depth discussion of the concepts presented in this paper as well as for conveying the critiques and concerns of experts in the SRF community. I especially acknowledge Ilan Ben-Zvi for bringing to my attention the importance of reducing FM fields in the location of vacuum flanges in SRF linacs. I also would like to thank Rama Calaga (BNL/CERN) and Jacek Sekutowicz (DESY) for their constructive criticism of the ideas presented here. This work is supported by Brookhaven Science Associates, LLC, under Contract No. DE-AC0298CH10886 with the U.S. Department of Energy.

[1] V.N. Litvinenko, in Proceedings of the 21st Particle Accelerator Conference, Knoxville, 2005 (IEEE, Piscataway, NJ, 2005), pp. 2768-2770.

[2] V. Ptitsyn et al., in Proceedings of the 10th European Particle Accelerator Conference, Edinburgh, Scotland, 2006 (EPS-AG, Edinburgh, Scotland, 2006), pp. 676-678.

[3] I. Ben-Zvi, in Proceedings of the 4th Asian Particle Accelerator Conference, Indore, 2007 (RRCAT, Indore, India, 2007), pp. 56-60.

[4] V. Ptitsyn et al., in Proceedings of the 23rd Particle Accelerator Conference, Vancouver, Canada, 2009 (IEEE, Piscataway, NJ, 2009).

[5] T. Kasuga et al., in Proceedings of the 4th Asian Particle Accelerator Conference, Indore, 2007 (Ref. [3]), pp. 172174. 
[6] G.H. Hoffstaetter, in Proceedings of the ERL 2005 Workshop, Newport News, VA, USA, http://www.Ins. cornell.edu/ hoff/hoff/papers/ERL05-08.pdf.

[7] G. H. Hoffstaetter et al., in Proceedings of the ERL 2007 Workshop, Daresbury, England, http://www.lns.cornell. edu/ hoff/hoff/papers/ERL05-08.pdf.

[8] M. Borland et al., Nucl. Instrum. Methods Phys. Res., Sect. A 582, 54 (2007).

[9] G. R. Neil et al., Nucl. Instrum. Methods Phys. Res., Sect. A 557, 9 (2006).

[10] G. R. Neil and L. Merminga, Rev. Mod. Phys. 74, 685 (2002).

[11] V.N. Litvinenko et al., in Proceedings of the 26th International FEL Conference, Trieste, Italy, 2004 (Comitato Conferenze Elettra, Trieste, 2004), pp. 594597.

[12] M. Sawamura et al., in Proceedings of the 9th European Particle Accelerator Conference, Lucerne, 2004 (EPSAG, Lucerne, 2004), pp. 1723-1725.

[13] F. Zimmerman et al., in Proceedings of the 23rd Particle Accelerator Conference, Vancouver, Canada, 2009 (Ref. [4]).

[14] F. Zimmerman et al., in Proceedings of the 11th European Particle Accelerator Conference, Genoa, 2008 (EPS-AG, Genoa, Italy, 2008), pp. 2847-2849.

[15] I. Ben-Zvi, Ya. Derbenev, V.N. Litvinenko, and L. Merminga, Nucl. Instrum. Methods Phys. Res., Sect. A 557, 28 (2006).

[16] V. N. Litvinenko and Y. S. Derbenev, Phys. Rev. Lett. 102, 114801 (2009).

[17] I. Ben-Zvi et al., AIP Conf. Proc. 821, 75 (2006).

[18] Ya. Derbenev, J. Musson, and Y. Zhang, in Proceedings of COOL2007, Bad Kreuznach, Germany, 2007, http:// cool07.gsi.de/contribution/THAP12.pdf.

[19] A. V. Fedotov et al., New J. Phys. 8, 283 (2006).

[20] G. H. Hoffstaetter and I. V. Bazarov, Phys. Rev. ST Accel. Beams 7, 054401 (2004).

[21] J. J. Bisognano and R. L. Gluckstern, in Proceedings of the 1987 Particle Accelerator Conference, Washington, DC (1987), p. 1078.

[22] R.E. Rand, Recirculating Electron Accelerators (Har- wood Academic Publishers, New York, 1984), Sec. 9.5.

[23] K. Beard, L. Merminga, and B. C. Yunn, in Proceedings of the 20th Particle Accelerator Conference, Portland, OR, 2003 (IEEE, New York, 2003), p. 332.

[24] B. Barstow, M. Liepe, and H. Padamsee, Engineering a Light Source for the Future, http://srf2003.desy.de/fap/ paper/ThP20.pdf.

[25] J. Sekutowicz, in Proceedings of the Sixth Workshop on SRF, Newport News, Virginia, 1993.

[26] J. Sekutowicz, in Proceedings of the 20th Particle Accelerator Conference, Portland, OR, 2003 (Ref. [23]), p. 1575, http://accelconf.web.cern.ch/accelconf/p03/ PAPERS/TPPE013.pdf; J. Sekutowicz, P. Kneisel, G. Ciovati, and L. Xiao, in Proceedings of the XXIV Linear Accelerator Conference, 2008, Victoria, British Columbia, Canada, p. 885, http://accelconf.web.cern.ch/ accelconf/LINAC08/papers/thp044.pdf.

[27] Sang-ho Kim et al., in Proceedings of LINAC 2006, Knoxville, Tennessee, USA (2006), p. 770, http:// accelconf.web.cern.ch/AccelConf/106/PAPERS/THP081. PDF.

[28] R. A. Rimmer et al., in Proceedings of the 2007 Particle Accelerator Conference, Albuquerque, New Mexico (IEEE, Albuquerque, New Mexico, 2007), p. 2493.

[29] S. Belomestnykh and V. Shemelin, in Proceedings of the 12th International Workshop on RF Superconductivity (SRF2005), Ithaca, NY, 2005, http://www.lns.cornell. edu/public/SRF/2006/SRF060424-03/SRF060424-03.pdf.

[30] J.D. Jackson, Classical Electrodynamics (Wiley, New York, 1998), 3rd ed., Chap. 8.

[31] R. Calaga, Ph.D. thesis, Department of Physics and Astronomy, Stony Brook University, 2006

[32] H. Hahn et al., HOM Absorbers for ERL Cryomodules at BNL (unpublished).

[33] R. Calaga, in Proceedings of SRF2009, Berlin, Germany, 2009, p. 515, http://srf2009.bessy.de/papers/thppo003.pdf.

[34] R. Calaga et al., Nucl. Instrum. Methods Phys. Res., Sect. A 557, 243 (2006).

[35] R. Calaga, thesis, SUNY, Stony Brook, NY, USA, 2006.

[36] I. Ben Zvi (private communication). 